\title{
Erratum to: Ongoing Response in BRAF V600E-Mutant Melanoma After Cessation of Intermittent Vemurafenib Therapy: A Case Report
}

\author{
Andrew J. Dooley ${ }^{2} \cdot$ Avinash Gupta $^{1} \cdot$ Mark R. Middleton $^{1}$
}

Published online: 28 March 2017

(C) Springer International Publishing Switzerland 2017

\section{Erratum to: Targ Oncol (2016) 11:557-563}

DOI 10.1007/s11523-015-0410-9

The article Ongoing Response in BRAF V600E-Mutant Melanoma After Cessation of Intermittent Vemurafenib Therapy: A Case Report, written by Andrew J. Dooley, Avinash Gupta and Mark R. Middleton, was originally published Online First without open access. After publication in volume 11 , issue 4 , page 557-563 the author decided to opt for Open Choice and to make the article an open access publication. Therefore, the copyright of the article has been changed to (C) The Author(s) 2016 and the article is forthwith distributed under the terms of the Creative Commons Attribution 4.0 International License (http://creativecommons.org/licenses/by/4.0/), which permits use, duplication, adaptation, distribution and reproduction in any medium or format, as long as you give appropriate credit to the original author(s) and the source, provide a link to the Creative Commons license, and indicate if changes were made.

The online version of the original article can be found at http://dx.doi.org/ 10.1007/s11523-015-0410-9.

Mark R. Middleton

mark.middleton@oncology.ox.ac.uk

1 Department of Oncology, NIHR Biomedical Research Centre, Oxford Cancer and Haematology Centre, Churchill Hospital, Old Road, Headington, Oxford OX3 7LE, UK

2 University of Oxford, John Radcliffe Hospital, Oxford OX3 9DU, UK 